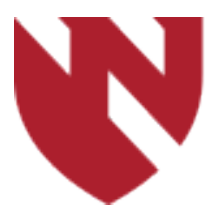

December 2020

\title{
A Case of Carcinoid Heart Disease and Echocardiographic Findings
}

\author{
Brandon Alex \\ University of Nebraska Medical Center \\ Jeffrey Songster \\ University of Nebraska Medical Center \\ HelenMari L. Merritt \\ Methodist Health System, Omaha, NE \\ Rebecca A. Aron \\ University of Nebraska Medical Center
}

Tell us how you used this information in this short survey.

Follow this and additional works at: https://digitalcommons.unmc.edu/gmerj

Part of the Anesthesiology Commons, Cardiology Commons, Higher Education Commons, and the Surgery Commons

\section{Recommended Citation}

Alex, B., Songster, J., Merritt, H. L., , Aron, R. A. A Case of Carcinoid Heart Disease and Echocardiographic Findings. Graduate Medical Education Research Journal. 2020 Dec 09; 2(2).

https://digitalcommons.unmc.edu/gmerj/vol2/iss2/6

This Case Report is brought to you for free and open access by DigitalCommons@UNMC. It has been accepted for inclusion in Graduate Medical Education Research Journal by an authorized editor of DigitalCommons@UNMC. For more information, please contact digitalcommons@unmc.edu. 


\title{
A Case of Carcinoid Heart Disease and Echocardiographic Findings
}

\author{
Abstract \\ regurgitation, tricuspid stenosis, pulmonic regurgitation and pulmonic stenosis.

\section{Keywords} \\ Carcinoid heart disease, echocardiographic findings in carcinoid \\ Creative Commons License \\ (c) $)(9)$
}

Abstract: Carcinoid heart disease occurs in approximately $50 \%$ of patients with carcinoid syndrome. Right heart involvement resulting in tricuspid regurgitation, tricuspid stenosis, pulmonic regurgitation and pulmonic stenosis is most common. We report a case of tricuspid heart disease resulting in tricuspid

This work is licensed under a Creative Commons Attribution-Noncommercial-No Derivative Works 4.0 License. 


\title{
A Case of Carcinoid Heart Disease and Echocardiographic Findings
}

\author{
Brandon R. Alex ${ }^{1}$, Jeffrey Songster ${ }^{1}$, HelenMari L. Merritt ${ }^{2}$, Rebecca A. Aron
}

'University of Nebraska Medical Center, Department of Anesthesiology, Omaha, NE

${ }^{2}$ Methodist Health System, Omaha, NE

\begin{abstract}
Carcinoid heart disease occurs in

approximately $50 \%$ of patients with carcinoid syndrome. Right heart involvement resulting in tricuspid regurgitation, tricuspid stenosis, pulmonic regurgitation and pulmonic stenosis is most common. We report a case of tricuspid heart disease resulting in tricuspid regurgitation, tricuspid stenosis, pulmonic regurgitation and pulmonic stenosis.
\end{abstract}

\section{Introduction}

Carcinoid tumors are rare $(1 / 75,000)$ neuroendocrine malignancies that typically arise from the appendix, terminal ileum, and other parts of the gastrointestinal tract and bronchopulmonary system. ${ }^{1}$ Carcinoid syndrome occurs in approximately $50 \%$ of patients and results in flushing, hypotension, diarrhea and/or bronchospasm. It occurs when tumors metastasize to the liver, allowing high levels of vasoactive hormones, such as serotonin, to reach beyond the portal circulation into the systemic circulation. Alternatively, primary ovarian or pulmonary carcinoid tumors may produce carcinoid syndrome without metastases. Approximately $50 \%$ of those with carcinoid syndrome develop carcinoid heart disease. ${ }^{1}$ Circulating vasoactive substances such as serotonin cause paraneoplastic effects, which impact the heart leading to valve fibrosis primarily affecting the right side of the heart, including the tricuspid valve and pulmonic valves. Right heart involvement is more commonly seen than left heart involvement, likely because of inactivation of vasoactive substances by the lungs. Right sided heart involvement presents with hepatomegaly, edema, ascites, fatigue, and low cardiac output. ${ }^{5}$ If left sided disease occurs, one should look for bronchial carcinoid (directed drainage into the left side), a patent foramen ovale (right to left shunt), or extensive liver metastases (overwhelms inactivation by the lungs). Verbal telephone consent was obtained from the patient for this case report.

\section{Case Presentation}

A 56-year-old female with ovarian neuroendocrine tumor and carcinoid heart disease presented for tricuspid valve (TV) replacement. Symptoms included dyspnea, lower extremity edema, and abdominal bloating. She previously underwent total hysterectomy, right salpingooophrectomy, cholecystectomy, appendectomy, omentectomy, and lymph node dissection with a curative intent. Transesophageal echo (TEE) revealed severely thickened and retracted TV leaflets, with severe tricuspid regurgitation (TR) and mild tricuspid stenosis (TS) (Figure 1). Although preoperative MRI reported mild pulmonic regurgitation (PR) and stenosis, intraoperative TEE revealed a PR pressure half time of $145 \mathrm{~ms}$ and a PR jet width/ pulmonic annulus ratio of 0.75 , suggesting moderate to severe PR (Figure 2). The TV leaflets were thickened and retracted, with a classic carcinoid appearance. The TV was replaced with a $27 \mathrm{~mm}$ St. Jude bioprosthesis as the patient preferred to avoid warfarin. A pulmonary arteriotomy was performed and the PV was examined, revealing mild leaflet thickening and a small annulus $(<19 \mathrm{~mm})$. Given concern that small size may limit future percutaneous procedures as well as the morbidity associated with dual right sided valve replacement, the PV was not replaced. Postprocedural TEE revealed a well seated TV without perivalvular leak, regurgitation, or significant gradient. The patient has been followed yearly and moderate to severe PR has persisted. Thus far her symptoms have improved, and the PR has been well tolerated.

\section{Discussion}

Carcinoid heart disease is a rare cause of TV and PV disease. The three-year survival rate is $31 \%$. Patients without cardiac involvement have a much better prognosis, with a survival rate nearly double those with cardiac involvement. ${ }^{1,2}$ Paraneoplastic effects of circulating serotonin promotes myofibrocytes to deposit plaques onto the endocardial surface of the right heart causing fibrosis. This results in leaflet thickening, retraction and subvalvular shortening, leading to regurgitation/stenosis primarily affecting the TV and PV. ${ }^{3}$ TR is most common (92$100 \%)$, followed by TS (38-44\%), PR (31-

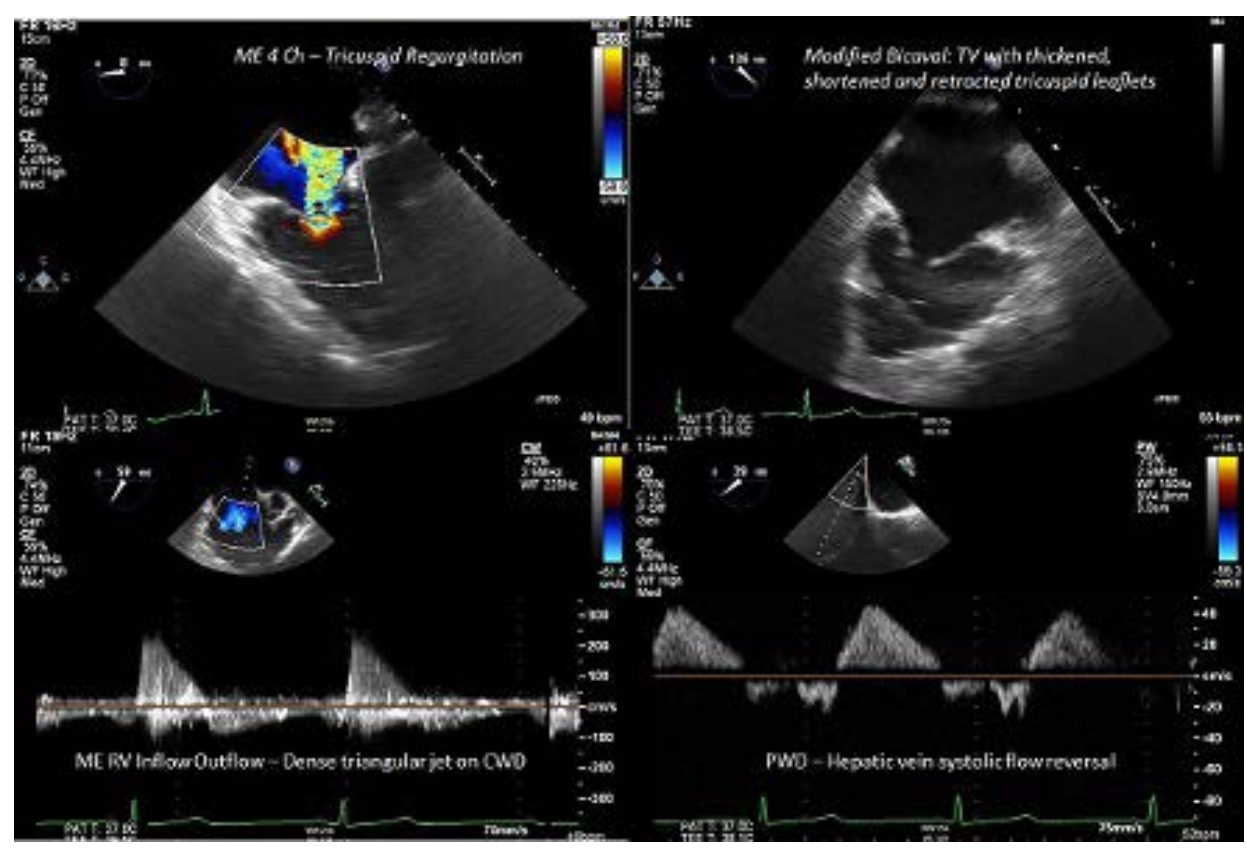

Figure 1. Tricuspid Valve Transesophageal Echo Findings: Midesophageal 4 chamber view showing severe tricuspid valvular regurgitation via color flow Doppler (top left). Modified bicaval view with thickened, shortened, and retracted tricuspid valve leaflets (top right). Mid esophageal right ventricular inflow outflow view with dense triangular jet via continuous wave Doppler suggesting severe tricuspid regurgitation (bottom left). Pulse wave doppler of hepatic vein with systolic flow reversal suggesting severe tricuspid regurgitation (bottom right). 


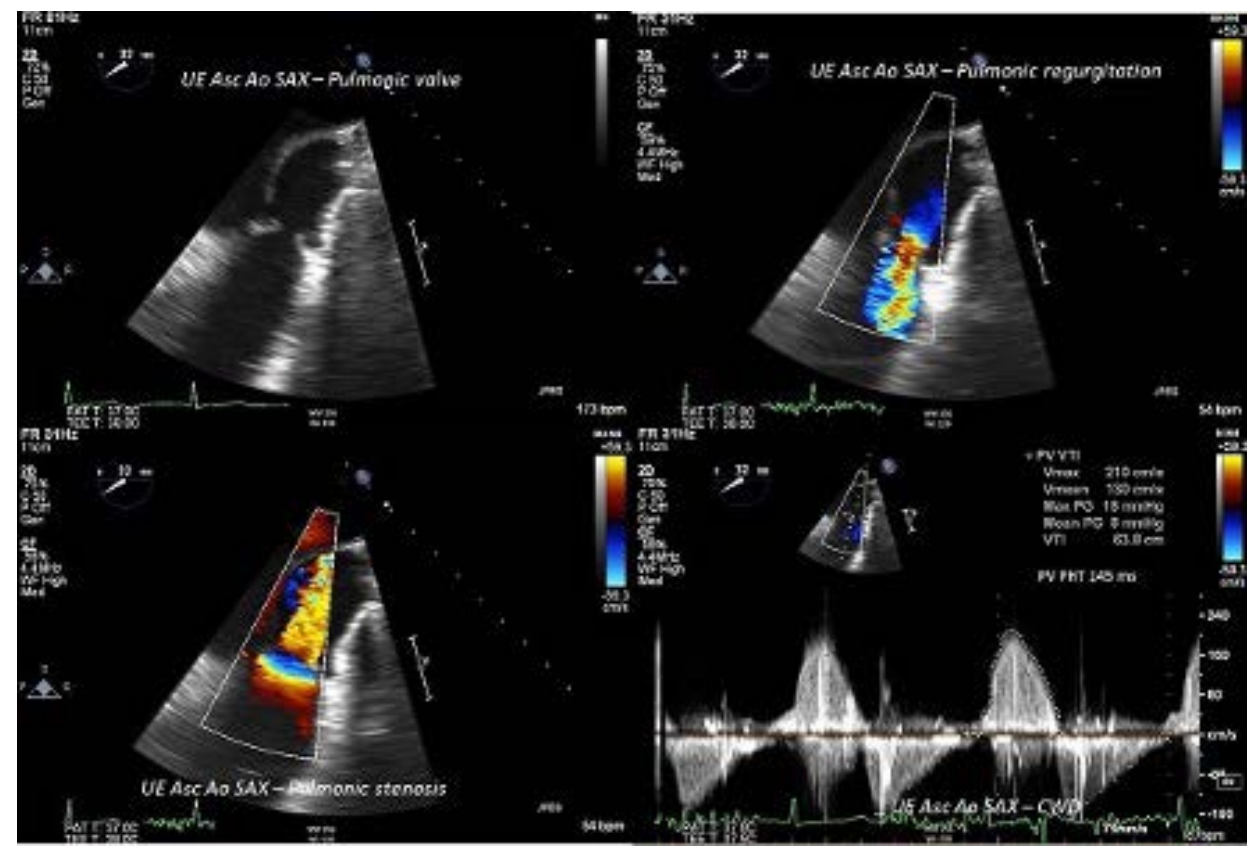

Figure 2. Pulmonic Valve Transesophageal Echo Findings: Upper esophageal ascending aorta short axis view showing malcoaptation of pulmonic valve (top left). Upper esophageal ascending aorta short axis view with severe pulmonic regurgitation via color flow doppler (top right). Upper esophageal ascending aorta short axis view with severe pulmonic stenosis via color flow doppler (bottom right). Upper esophageal ascending aorta short axis view with severe pulmonic stenosis via continuous wave doppler and mean gradient $8 \mathrm{mmHg}$ (lower right).

$38 \%)$ and pulmonic stenosis $(25-31 \%){ }^{2}$ Left heart valves are typically spared as the lungs inactivate the vasoactive substances. Dual valve replacement is technically challenging and has increased short and long-term morbidity and mortality compared to single valve replacement. ${ }^{1}$ Surgical management of the PV depends on the pathological extent and the patient's condition. PR may contribute to the development of RV dilatation and eventually RV dysfunction-induced heart failure, although rare. Transcatheter valves should be considered in high-risk candidates. ${ }^{4}$ Pulmonary valvectomy has been performed with varying outcomes including worsening right sided heart failure. ${ }^{4}$

\section{Conclusion}

This case illustrates the value of intraoperative echo to help guide the surgical approach to affected valves. Echo allows for the detailed analysis of coaptation zones, leaflet involvement, chamber assessment, and the subvalvular apparatus associated with each valve. ${ }^{5}$ Surgical treatment for severe hemodynamic consequences of carcinoid heart disease remains the mainstay of treatment to improve quality of life and longevity.

https://doi.org/10.32873/unmc.dc.gmerj.2.2.008

\section{References}

1 Fox DJ and Khattar RS. Carcinoid Heart Disease: Presentation, Diagnosis, and Management. Heart. 2004; 90: 1224-1228.

2 Connolly HM, Nishimura RA, Smith HC, et al. Outcome of Cardiac Surgery for Carcinoid Heart Disease. JACC. Feb 1995; 25 (2): 410-6.

3 Pellikka PA, Tajik JA, Khandheria BK, et al. Carcinoid Heart Disease: Clinical and Echocardiographic Spectrum in 74 Patients. Circulation. April 1993; 87 (4): 1188-1196.

4 Yuan, S. Valvular Disorders in Carcinoid Heart Disease. Brazilian Journal of Cardiovascular Surgery. Sept-Oct 2016; 31 (5):400-5

5 Fazlinezhad A, Moravvej Z, Azari A, Bigdelu L. Carcinoid heart disease and the utility of 3D transthoracic and trans-esophageal echocardiography: Two clinical cases. J Saudi Heart Assoc. 2014;26(1):51-55. doi:10.1016/j.jsha.2013.08.004 